\title{
Norois
}

Environnement, aménagement, société

$224 \mid 2012$

Agriculture paysanne, circuits courts, territoires périurbains

\section{Les représentations de l'activité agricole dans les projets de territoires périurbains : exemple de trois Pays en Midi-Pyrénées}

Representations of Farming in Exurban Development Projects: Three Case

Studies in Midi-Pyrénées (France)

Isabelle Duvernoy, Laurence Barthe et Stéphanie Lima

\section{(2) OpenEdition}

Journals

Édition électronique

URL : http://journals.openedition.org/norois/4273

DOI : $10.4000 /$ norois.4273

ISBN : 978-2-7535-2157-5

ISSN : $1760-8546$

Éditeur

Presses universitaires de Rennes

Édition imprimée

Date de publication : 30 septembre 2012

Pagination : 49-60

ISBN : $978-2-7535-2155-1$

ISSN : 0029-182X

Référence électronique

Isabelle Duvernoy, Laurence Barthe et Stéphanie Lima, « Les représentations de l'activité agricole dans les projets de territoires périurbains : exemple de trois Pays en Midi-Pyrénées », Norois [En ligne], 224 | 2012, mis en ligne le 30 septembre 2014, consulté le 30 avril 2019. URL : http:// journals.openedition.org/norois/4273 ; DOI : 10.4000/norois.4273 


\title{
Les représentations de l'activité agricole dans les projets de territoires périurbains : exemple de trois Pays en Midi-Pyrénées
}

\author{
Representations of Farming in Exurban Development Projects \\ Three Case Studies in Midi-Pyrénées (France)
}

Isabelle Duvernoy ${ }^{\mathrm{a} *}$, Laurence BARTHE ${ }^{\mathrm{b}}$, Stéphanie LiMA ${ }^{\mathrm{b}}$

\footnotetext{
* Auteur correspondant

a INRA, Centre de recherches de Toulouse, AGIR - UMR 1248 INRA INPT, BP 52627 - 31326 CASTANET-

Tolosan cedex, (dwvernoy@toulouse.inra.fr)

b Dynamiques Rurales - UMR MENRT 1936 MA 104 (Université de Toulouse - Le Mirail), 5, allée Antonio-

Machado-31058 Toulouse cedex 9 (barthe@univ-tlse2.fr) (Stephanie.Lima@wanadoo.fr)
}

Résumé : Pour étudier les représentations de l'activité agricole locale inscrites dans la construction des territoires de projet, nous proposons une grille de lecture systématisée de ces représentations s’inspirant de deux théories géographiques de la construction du territoire et de la territorialisation des activités. Nous mettons à l'épreuve cette grille dans trois Pays de Midi-Pyrénées, tous trois marqués par une forte périurbanisation. Le corpus est constitué des documents de constitution des Pays, de planification supracommunale (SCoT) et est complété par des entretiens auprès de différents acteurs territoriaux et agricoles. Cette classification fait apparaître une représentation différenciée de l'agriculture dans les projets de territoire, représentation parfois encore en cours d'élaboration. Un des cas étudiés suggère la mise en place de systèmes d'acteurs nouveaux appuyant le développement des activités agricoles suivant ces représentations.

\begin{abstract}
Representations of farming in exurban development projects. This paper results from an on-going research on the interconnections between rural development processes and farming development in the South of France. (Haute-Garonne and Tarn departments). We elaborate a tool to classify the links between farming and territorial development in territorial projects, according to two geographical theories of territoriality. We have analysed the representations of farming in three sample "Pays" (cross-municipality structures with participatory local development attributions, plus, in some cases, land planning attributions) in the vicinity of Toulouse, the $4^{\text {th }}$ largest city in France. These territories share the same twofold feature: a deep and increasing intertwining of urban development and rural land uses, and the preservation of their rural character as an identity label. In each case, we have examined the local territory project and operation documentation, and conducted interviews with the major actors in territorial planning and farming development institutions in the area. This research leads to a comparative assessment of the underlying creative thinking in each territorial project, and more particularly how farming activities and land use are included in the different territorial projects.
\end{abstract}

Mots clés : Périurbanisation, Activité agricole, Territoire, Représentations, Midi-Pyrénées

Keywords: Exurbanisation, Agriculture, Territory, Social representations, Midi-Pyrénées 


\section{INTRODUCTION}

Cet article porte sur la participation des territoires de projet à la définition d'une agriculture dont le développement est pensé comme articulé au développement des territoires. Nous nous différencions des travaux portant sur l'agriculture multifonctionnelle qui la décrivent comme le résultat de l'effet de politiques publiques de niveau général sur les décisions prises par des agriculteurs individuels. Nous cherchons au contraire à identifier le travail réalisé par des entités médiatrices, les territoires, pour donner forme à une multifonctionnalité définie en partie localement. Qualifier ces entités de «médiatrices» signifie que nous ne les concevons pas comme isolées des politiques publiques plus globales, régionales ou nationales.

En France, les «territoires de projet », comme par exemple les Pays (loi Voynet du 25 juin 1999), ont été conçus comme un nouvel espace de coordination, en capacité de contractualiser avec les autorités publiques pour mener à bien un projet de développement défini localement sur un périmètre pluri-communal. De plus, les Pays ont été pensés comme des entités porteuses de projets pluralistes, intersectoriels, s'appuyant sur une large représentation sociale (conseil de développement); la définition de leur périmètre devait favoriser l'articulation entre « urbain et rural ». Pour ces raisons, nous postulons que ces «territoires de projet " peuvent être en position d'assurer de nouvelles médiations entre l'activité agricole et la société, en articulant le développement agricole à d'autres champs d'activité publique ou privée que le secteur agricole. En outre, du fait de leur finalité de développement et de construction territoriale, ils peuvent être un lieu de reformulation des finalités de l'agriculture locale, notamment en lien avec leurs actions en termes de développement durable, suite notamment au Grenelle de l'environnement. Enfin, la première étape qui préside à leur création officielle est la proposition d'un périmètre justifié, qui est une première réflexion sur l'identité de ce territoire à partir de ses composantes et de leurs relations. La construction d'une identité territoriale va d'ailleurs bien au-delà de cette étape de délimitation.

Dans cet article, nous souhaitons examiner quelles représentations de l'activité agricole locale sont inscrites dans la construction territoriale des
Pays. Nous suivons là les propositions avancées par M. A. Hajer et H. Wagenaar (2004) pour l'analyse discursive des politiques publiques. Ces auteurs définissent un discours comme un ensemble d'idées, de concepts, de catégorisations qui soutiennent les pratiques. Les discours légitiment certaines pratiques et de ce fait certains acteurs. M. A. Hajer (2004) identifie notamment dans les discours des «story-lines ", qui condensent dans des images simples différents éléments auparavant épars et qui peuvent donc servir à former de nouvelles coalitions. Nous nous attacherons donc à discerner comment l'agriculture (appréhendée à travers des espaces, activités, acteurs ou institutions) constitue un élément des discours sur le territoire ou du projet territorial, et comment elle s'articule à d'autres composantes de ces mêmes discours.

Pour ce faire, nous proposons une grille de lecture systématisée qui doit permettre de comparer ces différentes représentations. Elle répond à un objectif pragmatique : pouvoir organiser et comparer les représentations de l'activité agricole de différents Pays et parcs naturels régionaux étudiés dans un même projet de recherche ${ }^{1}$. Elle répond également à un objectif finalisé de présentation des résultats de recherche mais aussi de mise en discussion de ceux-ci dans une forme qui permette de représenter la variété des trajectoires de développement pour l'activité agricole locale.

Pour construire une telle grille de lecture, nous nous appuyons principalement sur la théorie du territoire de deux géographes : G. Di Méo (1998) et J. Agnew (1990). Leurs travaux nous ont paru complémentaires car privilégiant deux dimensions différentes du territoire, la première abordant les domaines supports de la construction territoriale, du matériel à l'immatériel, la deuxième traitant des différentes façons dont une activité peut se territorialiser. Ainsi, notre grille de lecture croise deux points de vue : la construction du territoire et la construction par le territoire.

Pour G. Di Méo, la construction territoriale mobilise quatre niveaux en interaction.

Un niveau dit géographique, c'est-à-dire, dans sa pensée, plus matériel, qui comprend les paysages produits, les pratiques de l'espace mais également

1. Ce projet nommé « Agriculture et Gouvernance des Territoires » fait partie du programme de recherche PSDR3 Midi-Pyrénées et est co-financé par l'INRA et la Région Midi-Pyrénées, que nous remercions de leur soutien. 
les effets de structuration de l'espace notamment à travers la création d'échelons nouveaux et par le maillage qui est produit. Nous nous posons ainsi la question de la façon dont l'occupation agricole du sol est conçue, de sa place dans la structuration de l'espace (maillage, délimitation) et des paysages valorisés.

Un niveau plus économique comprend la sélection, l'organisation et la localisation des activités. C'est d'ailleurs là un élément prégnant et volontariste des projets de territoire portés par les Pays, en particulier là où ces derniers sont formés à partir des réflexions portées par les comités de bassin d'emploi. La localisation des activités, à travers la localisation des zones d'activités et des espaces productifs à vocation agricole, est également un élément important des démarches de planification urbaine supra-communale, telles que les SCoT.

Un niveau plus sociologique concerne les relations de pouvoir, les légitimités sur lesquelles s'appuie la construction territoriale, qui est le résultat à un moment donné de la supériorité ou de la légitimité d'un groupe d'acteurs, qui, en le façonnant s'approprie l'espace pour en faire le territoire de ses projets. Ce niveau sociologique, dans la présentation qu'en fait Di Méo (1998), est celui où les acteurs et leurs projets pour le territoire se confrontent ou s'associent. Nous le simplifions ici comme le niveau des relations des acteurs agricoles, individuels ou collectifs, entre eux ou avec d'autres.

Le niveau idéologique de la construction territoriale comprend les représentations qui soutiennent et guident la construction territoriale. C'est là que nous classerons les dimensions plus patrimoniales des représentations de l'activité agricole.

Les travaux de J. Agnew (1990) portent sur un point différent : ils permettent d'appréhender comment les activités s'inscrivent dans le territoire. En discutant les travaux d'A. Giddens, il différencie trois facettes de la territorialisation qu'il nomme, en français la localisation, le local et le «sens des lieux ».

La localisation se rapporte à l'emplacement de l'activité suivant des exigences d'ordre global (division du travail, système global de production). Ce n'est pas la qualité singulière du lieu qui détermine l'emplacement, ou les effets de cet emplacement, mais plutôt certaines de ses qualités génériques pour l'activité.
Le local, dans un sens proche de celui utilisé par A. Giddens, désigne le lieu comme un contexte d'interaction. Là, l'activité et les acteurs sont conçus comme reliés à d'autres activités et d'autres acteurs parce que partageant un même espace aménagé, où ils se rencontrent.

Le «sens des lieux » fait référence à une dimension plus identitaire du territoire. L'activité agricole est elle envisagée comme participant à la construction de l'identité territoriale? C'est un point particulièrement important dans les territoires de projet qui ont à justifier leur délimitation et leur cohérence lors de leur création et lors de la mise en concurrence des territoires (appels d'offre, demande de financements publics).

Nous nous proposons donc dans cet article d'utiliser la grille résultant du croisement de ces deux théories du territoire et de la territorialisation (tableau 1) pour rendre compte des représentations de l'articulation de l'activité agricole aux projets territoriaux des Pays à partir de trois exemples.

\section{Présentation des cas étudiés ET CONSTITUTION DU CORPUS ANALYSÉ}

\section{Présentation des trois pays étudiés}

Nous avons choisi d'étudier préférentiellement des Pays en position périurbaine : en Haute-Garonne, les Pays Tolosan, et du Sud Toulousain, inclus dans l'aire urbaine de Toulouse, et le Pays Vignoble Gaillacois, Bastides et Val Dadou (VGBVD) dans le Tarn (figure 1). Ce dernier, situé dans le couloir d'urbanisation (Frénot, 2009) qui relie Toulouse à Albi, est le premier Pays constitué dans le Tarn, avec une première préfiguration dès 2001 (Barreau et Taulelle, 2008). Sur le périmètre de ces trois Pays, des Schémas de Cohérence Territoriale (SCoT) sont en cours d'élaboration ou approuvés. Les deux démarches Pays et SCoT sont conduites par le même syndicat mixte dans le cas des Pays Sud Toulousain et VGBVD (tableau 2). Le périmètre du Pays Tolosan a doublé fin 2008 pour se rapprocher, sans être exactement similaire, de celui du SCoT du nord toulousain porté par un syndicat mixte indépendant du Pays. 


\begin{tabular}{|c|c|c|c|}
\hline $\begin{array}{c}\text { Type d'interaction agriculture/ } \\
\text { territoire de projet ou territoire } \\
\text { planifié }\end{array}$ & $\begin{array}{c}\text { Localisation } \\
\text { Activité conçue comme } \\
\text { insérée dans un secteur mais } \\
\text { isolée dans le territoire }\end{array}$ & $\begin{array}{c}\text { Local } \\
\text { Activités conçue comme en } \\
\text { interaction localement avec } \\
\text { d'autres activités }\end{array}$ & $\begin{array}{c}\text { Sens des lieux } \\
\text { Activité participant à l'identité } \\
\text { territoriale }\end{array}$ \\
\hline $\begin{array}{c}\text { Dimension matérielle } \\
\text { (occupation du sol) }\end{array}$ & & & \\
\hline $\begin{array}{c}\text { Dimension économique } \\
\text { (production) }\end{array}$ & & & \\
\hline $\begin{array}{c}\text { Dimension sociale } \\
\text { (acteurs de l'activité et leurs } \\
\text { relations) }\end{array}$ & & & \\
\hline $\begin{array}{c}\text { Dimension idéologique } \\
\text { (activité patrimoniale, activité } \\
\text { participant à l'attractivité du } \\
\text { territoire) }\end{array}$ & & & \\
\hline
\end{tabular}

Tableau 1 : Grille d'articulation de l'activité agricole aux projets territoriaux Matrix of interactions between farming and rural development projects.

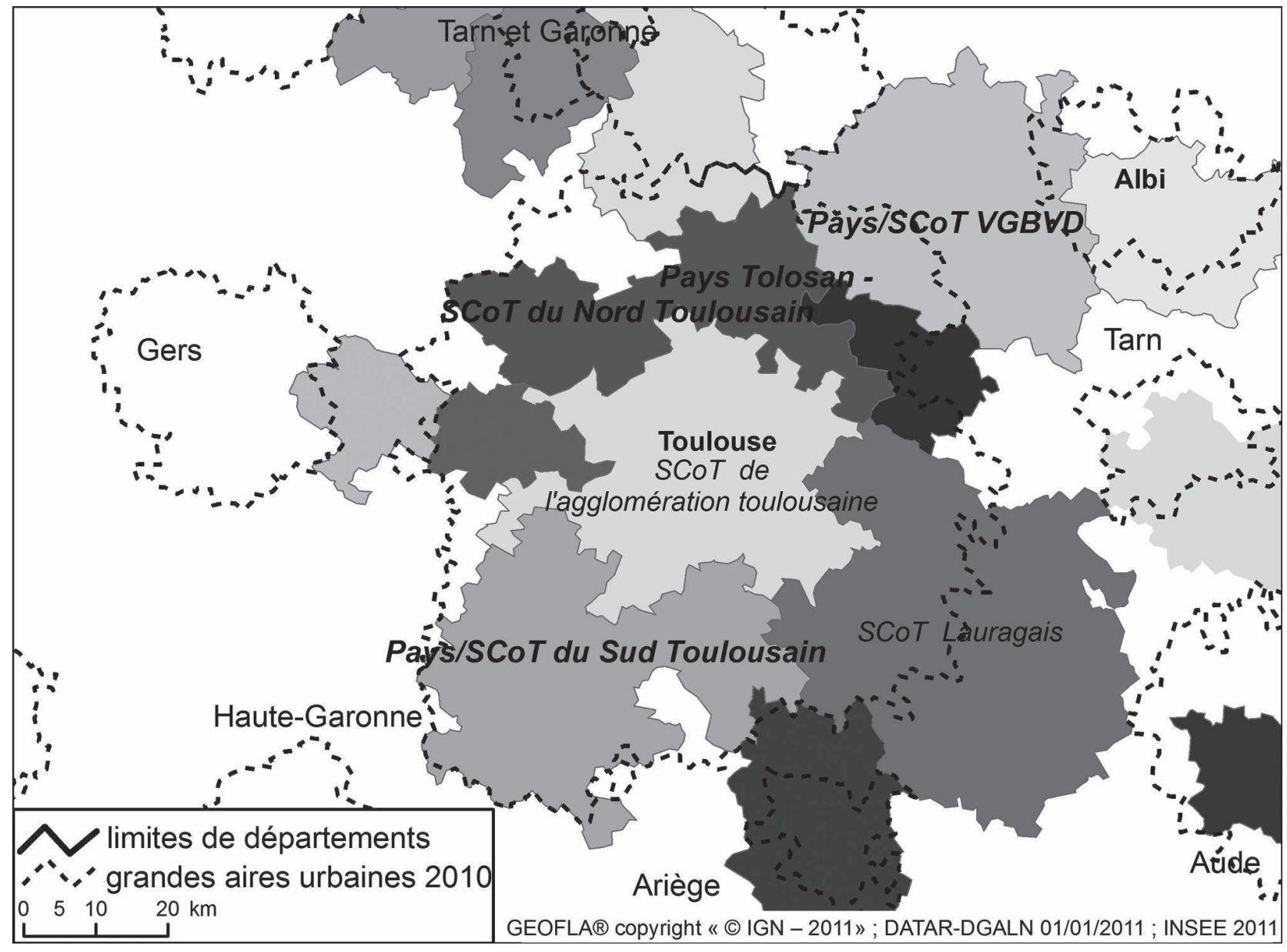

Figure 1 : Localisation des trois Pays étudiés par rapport aux périmètres des SCoT en 2011

Location of the three Pays under study in the structure plans (SCoT) map of the Midi-Pyrénées Region in 2011 


\begin{tabular}{|c|c|c|c|c|c|}
\hline $\begin{array}{c}\text { Pays } \\
\text { (date de création) }\end{array}$ & $\begin{array}{c}\text { Composition com- } \\
\text { munale } \\
\text { (nombre de } \\
\text { communautés de } \\
\text { communes) }\end{array}$ & $\begin{array}{l}\text { Population } 2006 \\
\text { (variation annuelle } \\
\text { de la population de } \\
1999 \text { à } 2006 \text { en \%) }\end{array}$ & $\begin{array}{c}\text { Nombre } \\
\text { d'exploitations agri- } \\
\text { coles en } 2000\end{array}$ & $\begin{array}{c}\text { Distance aux prin- } \\
\text { cipales aggloméra- } \\
\text { tions }\end{array}$ & $\begin{array}{c}\text { Structure Pays et } \\
\text { SCoT }\end{array}$ \\
\hline $\begin{array}{l}\text { Pays Tolosan } \\
(2002)\end{array}$ & $\begin{array}{l}68 \text { communes } \\
\text { (5 CC) }\end{array}$ & $\begin{array}{c}90426 \text { hab. } \\
(+2,7 \%)\end{array}$ & 1385 & $\begin{array}{l}\text { Toulouse } \\
(10 \mathrm{~km})\end{array}$ & $\begin{array}{c}\text { Association de Pays } \\
\text { et Syndicat Mixte } \\
\text { SCoT }\end{array}$ \\
\hline $\begin{array}{c}\text { Pays du Sud Tou- } \\
\text { lousain } \\
(2003)\end{array}$ & $\begin{array}{l}99 \text { communes } \\
\text { (6 CC) }\end{array}$ & $\begin{array}{l}80805 \text { hab. } \\
(+2,7 \%)\end{array}$ & 1761 & Toulouse (20 km) & $\begin{array}{l}\text { Syndicat Mixte por- } \\
\text { tant les procédures } \\
\text { Pays et SCoT }\end{array}$ \\
\hline $\begin{array}{l}\text { Pays VGBVD } \\
(2002)\end{array}$ & $\begin{array}{l}64 \text { communes } \\
\quad(4 \mathrm{CC})\end{array}$ & $\begin{array}{c}63805 \text { hab. } \\
(+1,2 \%)\end{array}$ & 1827 & $\begin{array}{l}\text { Toulouse }(30 \mathrm{~km}), \\
\text { Albi }(12 \mathrm{~km}), \text { Mon- } \\
\text { tauban }(24 \mathrm{~km})\end{array}$ & $\begin{array}{c}\text { Syndicat Mixte por- } \\
\text { tant les procédures } \\
\text { Pays et SCoT }\end{array}$ \\
\hline
\end{tabular}

Tableau 2 : Présentation succincte des trois Pays étudiés. (Source : Insee, portraits de territoires)

Rapid presentation of the three Pays under study

Ces trois Pays sont fortement marqués par la croissance démographique toulousaine, qui explique une grande partie de leur dynamique démographique rapide (tableau 2). Leur création répond à une volonté d'organiser cette croissance et de veiller à la qualité des retombées que ces territoires peuvent en attendre. Au nord de la Haute-Garonne, l'engagement dans des SCoT indépendants correspond au même souci, alors que l'Etat proposait un SCoT unique pour l'ensemble de l'aire urbaine de Toulouse. Néanmoins, les quatre SCoT en «marguerite » qui couvrent l'aire urbaine de Toulouse (figure 1) sont réunis dans un groupement d'intérêt public (GIP) «interscot » qui veille à la cohérence de leurs choix de développement urbain.

La création des Pays Tolosan et VGBVD s'est appuyée sur des expériences préalables de développement local intercommunal (comité de bassin d'emploi dans le premier cas, contrat « Espace 2000 » dans le deuxième).

\section{Corpus et grille d'analyse}

Pour chacun des cas étudiés, nous avons constitué le corpus de façon similaire. Celui-ci est constitué des documents officiels scandant la construction territoriale du Pays (charte, convention territoriale) et des documents préparatoires à l'établissement du SCoT (pré-PADD notamment). C'est sur ces documents que nous allons tester la grille proposée.

Ce corpus a été complété par différents entretiens semi-directifs auprès des personnes en charge de la construction territoriale (chargés de mission et élus territoriaux) ainsi qu'auprès des acteurs départementaux du développement agricole, (chambres d'agriculture de Haute-Garonne et du Tarn, services en charge des procédures d'aménagement du territoire), avec des représentants locaux des agriculteurs et avec les responsables des antennes locales des chambres d'Agriculture. Ces différents entretiens, menés en 2008 et 2009, portaient sur la façon dont l'activité agricole peut s'articuler à la construction territoriale. Ces entretiens ne seront mobilisés que ponctuellement dans ce travail pour apporter des éclairages complémentaires sur les informations recueillies dans les documents officiels.

\section{REPRÉSENTATIONS DE L'AGRICULTURE DANS LA CONSTRUCTION DES TERRITOIRES}

\section{Le Pays Tolosan}

Nous présentons l'analyse de la charte du Pays (2003) mais rappellerons quelques éléments de l'analyse du pré-PADD du SCoT (2007), qui est un document de planification spatiale.

L'agriculture est citée en plusieurs points de la charte du Pays : dans le Diagnostic de territoire, comme dans la partie décrivant le projet nommée Enjeux et axes de développement. Elle apparaît également en lien avec d'autres problématiques du territoire, le cadre de vie et l'environnement, le développement économique.

Dans le diagnostic, la périurbanisation paraît un trait caractéristique du Pays et de ses axes de 
réflexion. Dans ce sens, l'agriculture est évoquée généralement comme contribuant à ce trait, à travers l'image de «campagne » proche de la ville, ce qui pose évidemment le problème d'une concurrence pour l'usage du sol entre habitat et agriculture. Ce sont surtout la forêt et la vigne qui sont relevées comme éléments paysagers végétaux intéressants et apportant une spécificité au territoire. L'activité agricole est d'abord évoquée en lien avec la qualité des cours d'eau (pollution par les nitrates), et de façon indirecte dans « les dégradations paysagères » avec « lotissements mal intégrés aux centres villageois, apparitions de friches, remembrement des terres agricoles, création de hangars agricoles... ».

Se dessine dans le diagnostic une différenciation entre la viticulture (AOC Fronton, $20 \%$ des exploitants du Pays), dont la dimension patrimoniale est soulignée ("vignoble qui date du quatrième siècle ", future maison des vins), et les autres productions, moins décrites car peut-être jugées plus banales, et sans doute moins connues des porteurs du projet de Pays. La viticulture occupe une place modeste dans la superficie du territoire (tableau 3), mais elle est mobilisée par les acteurs du développement territorial comme une image symbolique, un emblème de l'agriculture territorialisée s'appuyant sur une AOC. La viticulture figure en haut lieu dans les discours et les représentations du territoire à la fois pour incarner des savoir-faire anciens, liés au terroir, garants d'une préservation de l'espace et pour promouvoir l'attractivité du territoire au regard des populations extérieures, futurs résidents et touristes. La viticulture a d'ailleurs été l'emblème mobilisateur dans le cadre d'un vaste mouvement de lutte des populations locales et de leurs élus face au projet d'implantation d'un aéroport dans ce territoire au début des années 2000.

\begin{tabular}{|l|c|c|c|}
\hline Pays & $\begin{array}{c}\text { Grandes } \\
\text { cultures }\end{array}$ & $\begin{array}{c}\text { Viticulture, } \\
\text { horticulture, } \\
\text { maraîchage }\end{array}$ & Élevage \\
\hline Pays Tolosan & $89 \%$ & $7 \%$ & $4 \%$ \\
\hline $\begin{array}{l}\text { Pays du Sud } \\
\text { Toulousain }\end{array}$ & $83 \%$ & $0 \%$ & $16 \%$ \\
\hline Pays VGBVD & $61 \%$ & $23 \%$ & $16 \%$ \\
\hline
\end{tabular}

Tableau 3 : Proportion en surface des grands types de productions agricoles dans les trois Pays en 2000. (Source : Insee, portraits de territoires)

Main types of productions as a percentage of total farm lands in the three Pays in 2000
Dans les enjeux et les axes de développement des éléments du diagnostic sont repris dans une perspective d'actions à entreprendre pour maintenir une activité agricole : favoriser la reprise des exploitations, limiter le mitage en préservant des zones agricoles dans les documents d'urbanisme. La diversité des productions est cette fois-ci soulignée, et le développement de circuits courts fortement mis en avant pour « développer la communication entre néo-ruraux et agriculteurs ». La préservation de l'environnement est également citée à travers le thème de l'agriculture raisonnée, des contrats d'agriculture durable. Un autre signe de la différenciation entre viticulture et autres productions, est donné par l'importance accordée à la seule viticulture dans les chapitres qui ne portent pas directement sur l'agriculture, notamment celui portant sur le tourisme de proximité.

On retrouve un même traitement différencié de la viticulture par rapport aux autres productions agricoles dans le pré-PADD du SCoT. À la viticulture est reconnu un rôle dans la construction de l'image du territoire à travers des actions emblématiques (route des vins, maison de vins). Le reste de l'agriculture est l'objet d'un regard plus classique : il s'agit d'améliorer les filières, la qualité des productions, d'adopter des pratiques plus respectueuses de l'environnement, de favoriser les circuits courts. Le Pays Tolosan a récemment mis en place un répertoire des producteurs locaux commercialisant en vente directe.

Nos observations et les entretiens indiquent la concrétisation depuis 2009 de nombreuses initiatives que le Pays peinait à rassembler, même si elles étaient a priori conformes au projet territorial : création d'un magasin de producteurs conduit par un conseiller agricole du Conseil Général, projet d’installation de maraîchers, réflexion sur des activités de chimie verte, portés par une communauté de communes à l'ouest du Pays... Les incertitudes sur le périmètre du Pays, qui n'englobe que depuis très récemment l'ensemble du périmètre couvert par le SCoT Nord Toulousain peut expliquer aisément que des projets concernant une partie du territoire qui ne sera rattachée que tardivement à la construction du Pays n'aient pas été articulés avec celle-là. On constate néanmoins dans les entretiens une passagère et faible représentation des agriculteurs (soit directe, soit à travers leurs représentants) dans les instances du Pays (la commission traitant d'agriculture ne s'est réunie que deux fois de 2004 à 2009). 
La question se pose donc du rôle que peut jouer le Pays dans l'articulation entre activité agricole et autres activités du territoire, et ce d'autant plus que l'élaboration du SCoT, et donc la localisation des terres qui se maintiendront comme terres agricoles, dépend d'une autre entité. Il n'en reste pas moins que le Pays a réussi à formuler une fonction agricole centrée sur la production de services à destination de la population locale et des touristes. Deux actions peuvent être mentionnées pour incarner cette orientation. La création de la maison des vins de Fronton qui rassemble les professionnels agricoles et les acteurs du tourisme autour de la promotion du produit emblématique qu'est le vin de Fronton. Par ailleurs, le Pays est à l'origine de la création d'une balade gourmande qui fédère tous les producteurs locaux engagés dans des démarches de vente directe et/ou d'accueil de consommateurs. L'agriculture est envisagée comme un élément de l'attractivité territoriale, d'un certain art de vivre, même si pour cela un processus de sélection est opéré dans la caractérisation de l'agriculture locale : mise en exergue de la viticulture et des productions de qualité; l'agriculture occupant la majeure partie du territoire restant en second plan (tableau 4).

\section{Le Pays Sud-Toulousain}

Dans le Pays Sud Toulousain, un seul syndicat mixte gère conjointement la mise en œuvre du projet de Pays et l'élaboration du SCoT. Les deux démarches sont donc associées. Pour comprendre les représentations en cours de l'activité agricole sur le territoire, nous analysons donc le document préparatoire du SCoT, le pré PADD (2007), que nous complétons par différents documents concernant les actions du Pays.

Deux types d'agriculture sont décrits comme à valoriser sur le territoire, en relation avec deux types d'espaces agricoles à protéger. Il s'agit d'une part des vallées, où les exploitations agricoles se spécialisent vers les grandes cultures, liées à des industries agro-alimentaires, menacées par la progression de l'urbanisation et, d'autre part, des coteaux, moins menacés par l'urbanisation, plus diversifiés dans les productions, même si l'élevage régresse, où pourrait se développer une agriculture raisonnée ou biologique tournée vers la diversification, le développement de circuits courts. On peut noter qu'au-delà de la question foncière, classique, le pré-PADD mentionne une attention à porter à l'installation en agriculture. Dans ce document, nous n'identifions pas nettement une participation de l'agriculture à la construction de l'identité du territoire (tableau 5).

Cette présentation de l'agriculture se retrouve à différents moments de la construction territoriale. En nous appuyant sur les entretiens et les observations de réunion, il nous semble qu'on peut parler de la construction d'une représentation duelle de l'activité agricole sur le territoire. Ces entretiens nous permettent de faire l'hypothèse d'un travail de construction d'une nouvelle catégorie d'activité

\begin{tabular}{|c|c|c|c|}
\hline $\begin{array}{c}\text { Type d'interaction agriculture/ } \\
\text { territoire de projet ou territoire } \\
\text { planifié }\end{array}$ & $\begin{array}{c}\text { Localisation } \\
\text { Activité conçue comme } \\
\text { insérée dans un secteur mais } \\
\text { isolée dans le territoire }\end{array}$ & $\begin{array}{c}\text { Local } \\
\text { Activités conçue comme en } \\
\text { interaction localement avec } \\
\text { d'autres activités }\end{array}$ & $\begin{array}{c}\text { Sens des lieux } \\
\text { Activité participant à l'identité } \\
\text { territoriale }\end{array}$ \\
\hline $\begin{array}{c}\text { Dimension matérielle } \\
\text { (occupation du sol) }\end{array}$ & $\begin{array}{c}\text { Concurrence agriculture/ } \\
\text { habitat }\end{array}$ & $\begin{array}{c}\text { Campagne, Paysage viticole } \\
\text { Pollution, pb paysagers. Agri- } \\
\text { culture raisonnée }\end{array}$ & Vignoble très ancien \\
\hline $\begin{array}{c}\text { Dimension économique } \\
\text { (production) }\end{array}$ & $\begin{array}{c}\text { Productions. Mise en avant de } \\
\text { la viticulture }\end{array}$ & $\begin{array}{c}\text { Valoriser, transformer com- } \\
\text { mercialiser en circuit court les } \\
\text { productions }\end{array}$ & \\
\hline $\begin{array}{c}\text { Dimension sociale } \\
\text { (acteurs de l'activité et leurs } \\
\text { relations) }\end{array}$ & $\begin{array}{c}\text { Transmission, main d'œuvre } \\
\text { Dimension idéologique } \\
\text { (activité patrimoniale, activité } \\
\text { participant à l'attractivité du } \\
\text { territoire) }\end{array}$ & $\begin{array}{c}\text { Vignoble produit d'appel pour } \\
\text { d'autres activités agritouris- } \\
\text { tiques }\end{array}$ & Maison des vins comme \\
vitrine
\end{tabular}

Tableau 4 : Représentations de l'articulation des activités agricoles au territoire dans la charte du Pays Tolosan Matrix of interactions between farming and rural development in the charte du Pays Tolosan 


\begin{tabular}{|c|c|c|c|}
\hline $\begin{array}{c}\text { Type d'interaction agriculture/ } \\
\text { territoire de projet ou territoire } \\
\text { planifié }\end{array}$ & $\begin{array}{c}\text { Localisation } \\
\text { Activité conçue comme } \\
\text { insérée dans un secteur mais } \\
\text { isolée dans le territoire } \\
\text { Difficultés dues à l'urbanisa- } \\
\text { tion des vallées }\end{array}$ & $\begin{array}{c}\text { Local } \\
\text { Activités conçue comme en } \\
\text { interaction localement avec } \\
\text { d'autres activités }\end{array}$ & $\begin{array}{c}\text { Sens des lieux } \\
\text { Activité participant à l'identité } \\
\text { territoriale }\end{array}$ \\
\hline $\begin{array}{c}\text { Dimension matérielle } \\
\text { (occupation du sol) }\end{array}$ & $\begin{array}{c}\text { Grandes cultures } \\
\text { (production) }\end{array}$ & $\begin{array}{c}\text { Grandes cultures (en lien avec } \\
\text { les IAA) }\end{array}$ & \\
\hline $\begin{array}{c}\text { Dimension économique } \\
\text { Dimension sociale } \\
\text { (acteurs de l'activité et leurs } \\
\text { relations) }\end{array}$ & $\begin{array}{c}\text { Circuits courts (en lien avec } \\
\text { activités commer- ciales, } \\
\text { artisanales) }\end{array}$ & \\
\hline $\begin{array}{c}\text { Dimension idéologique } \\
\text { (activité patrimoniale, activité } \\
\text { participant à l'attractivité du } \\
\text { territoire) }\end{array}$ & & & \\
\hline
\end{tabular}

Tableau 5 : Représentations de l'articulation des activités agricoles au territoire dans le pré-PADD du Pays du Sud Toulousain Matrix of interactions between farming and rural development in the pré-PADD du Pays Sud Toulousain

agricole (et d'agriculteurs) à partir d'un travail de formulation permettant d'englober des productions diverses. L'analyse de l'entretien réalisé avec un agent du Pays en charge de l'agriculture (et de l'environnement) permet de voir ce travail encore à l'œuvre. En suivant D. Demazière et C. Dubar (1997), nous avons tenté d'identifier les oppositions qui structurent les discours décrivant l'activité agricole dans le Pays. Si les termes eux-mêmes peuvent varier, une opposition est faite entre « céréaliers", « intensifs » et un autre type d'agriculteurs : « des agriculteurs qui veulent travailler de façon différente. ». C'est finalement le terme « agriculture durable » qui va servir à réunir ce dernier ensemble d'agriculteurs, en proposant une identité collective sur laquelle pourrait s'articuler la mise en œuvre de politiques publiques (Hajer, 2004).

La réflexion et les actions du Pays sur le maintien d'une activité agricole sont organisées en partie par cette opposition entre deux types d'activités. En effet, c'est en plaine que sont l'urbanisation et les réseaux de transport et où la concurrence foncière entre agriculture et urbanisation a été la plus forte, et où le pré-PADD du SCoT prévoit de structurer l'urbanisation. En revanche, moins accessibles, les coteaux sont moins soumis à la pression urbaine. Hormis la préservation de terres agricoles (en évitant le mitage, en organisant l'urbanisation) qui peut être inscrite dans le SCoT, le Pays a concentré ses actions sur le développement d'un «projet d'agriculture durable » (répertoire de producteurs, tentative d'organisations collective, participation à des réu- nions publiques sur l'installation en agriculture en mettant l'accent sur certaines formes d'installation (hors cadre familial) et certaines productions).

Pour reprendre le fil proposé par M. A. Hajer (2004), les discours qui s'organisent et organisent la mise en œuvre des politiques publiques peuvent favoriser la création de coalitions nouvelles d'acteurs. Nous faisons l'hypothèse que la distinction de deux agricultures au sein du Pays, peut conduire à terme à différencier deux systèmes d'action supportant l'agriculture dans le territoire. Les réunions publiques dédiées à l'agriculture (Tables rondes agricoles du Pays du Sud Toulousain) montrent l'intervention de nouveaux acteurs sur des questions agricoles en Haute-Garonne, comme la FD CIVAM et une association environnementale. Les acteurs plus classiques en Haute-Garonne de l'accompagnement agricole (Duvernoy, 2009), chambre d'Agriculture et Conseil général ne sont pas absents. Leur lien avec la construction des Pays paraît cependant assez ténu. Ces deux organismes sont impliqués dans la procédure SCoT, la chambre d'Agriculture en tant que personne publique associée, le Conseil général par l'assistance technique de son agence technique départementale. C'est donc par le biais du suivi de la planification territoriale (SCoT), que les organismes les plus impliqués dans le conseil agricole, sont d'abord présents dans la construction territoriale du Pays. 


\section{Le Pays Vignoble Gaillacois Bastides et Val Dadou}

Dans la convention territoriale (2008) du Pays, le développement urbain est présenté comme une dynamique «naturelle » du territoire, qui se positionne comme un «Pays d'accueil sous influence des aires urbaines dont l'attractivité est renforcée par la présence d'un patrimoine identitaire riche ». Dans la partie diagnostic du document, l'agriculture est évoquée comme étant menacée par la «résidentialisation » du territoire. Elle est citée aussi dans le cadre de la préservation des ressources locales et de la production d'énergie. En outre, l'activité agricole tient une place à part entière dans le diagnostic économique dans la mesure où elle représente 5000 emplois et une valeur identitaire marquée à travers ses espaces, plus particulièrement ceux dédiés à la viticulture (vignoble AOC Gaillac), sans oublier le potentiel que représente la ressource en bois. La principale faiblesse identifiée concerne la diminution de la surface agricole et la concentration des exploitations. En termes d'opportunités, la diversification de l'agriculture vers le tourisme et la vente directe (productions de qualité) est mise en avant. La principale menace identifiée concerne la perte des emplois agricoles et le risque de banalisation des paysages (« points noirs » paysagers, friches agricoles).

Occupant une place particulière dans le paysage économique du Pays, le vignoble fait l'objet d'une partie spécifique dans le diagnostic territorial. Trois indicateurs résument ses atouts : 3000 emplois,
7000 hectares et 18 millions de bouteilles d'appellation Gaillac par an. Au-delà de ces chiffres, le vignoble est aussi reconnu par le patrimoine culturel et la valeur identitaire qu'il représente. Le développement de l'œnotourisme et la production de qualité sont identifiés comme les principales opportunités. Les menaces concernent la nécessité de restructurer la filière (arrachages possibles).

On retrouve une structuration des représentations proche de celle déjà rencontrée dans le Pays Tolosan, en raison du poids dans les deux Pays de la viticulture en AOC (tableau 6). Cependant, dans le Pays VGBVD, la démarche est orientée de façon plus affirmée vers l'attractivité touristique du territoire, qui oriente une partie des actions identifiées au-delà de la sphère viticole (« aménagement de points d'accueil et de vitrines de produits à la ferme ", « investissement d'accueil à la ferme »).

Le SCoT du Pays, quant à lui, se veut un «SCoT rural », reposant sur une « inversion du regard » : partir des espaces ouverts, agricoles et naturels, pour tenter de les préserver. Cette attention a permis d'engager une réflexion sur une consommation économe de l'espace et sur les circulations agricoles dans le territoire. Elle rejoint celle de la chambre d'agriculture du Tarn, au sein de laquelle un service urbanisme a été structuré avec l'embauche d'un juriste spécialisé en droit de l'urbanisme. Ce dernier suit notamment l'ensemble des procédures d'aménagement du département, à différentes échelles. Le consensus sur une préservation des surfaces agricoles peut-il déboucher sur la construction commune d'un projet agricole pour le territoire?

\begin{tabular}{|c|c|c|c|}
\hline $\begin{array}{c}\text { Type d'interaction agriculture/ } \\
\text { territoire de projet ou territoire } \\
\text { planifié }\end{array}$ & $\begin{array}{c}\text { Localisation } \\
\text { Activité conçue comme } \\
\text { insérée dans un secteur mais } \\
\text { isolée dans le territoire }\end{array}$ & $\begin{array}{c}\text { Local } \\
\text { Activités conçue comme en } \\
\text { interaction localement avec } \\
\text { d'autres activités }\end{array}$ & $\begin{array}{c}\text { Sens des lieux } \\
\text { Activité participant à l'identité } \\
\text { territoriale }\end{array}$ \\
\hline $\begin{array}{l}\text { Dimension matérielle } \\
\text { (occupation du sol) }\end{array}$ & & $\begin{array}{l}\text { Risques de banalisation du } \\
\text { paysage }\end{array}$ & \\
\hline $\begin{array}{l}\text { Dimension économique } \\
\text { (production) }\end{array}$ & & \multirow[b]{2}{*}{ Emploi dans la filière viticole } & \\
\hline $\begin{array}{c}\text { Dimension sociale } \\
\text { (acteurs de l'activité et leurs } \\
\text { relations) }\end{array}$ & & & $\begin{array}{l}\text { Lien viticulture et diversifica- } \\
\text { tion agricole -tourisme }\end{array}$ \\
\hline $\begin{array}{c}\text { Dimension idéologique } \\
\text { (activité patrimoniale, activité } \\
\text { participant à l'attractivité du } \\
\text { territoire) }\end{array}$ & & $\begin{array}{c}\text { Entretien du paysage } \\
\text { Développement de circuits } \\
\text { courts }\end{array}$ & Oeno-tourisme \\
\hline
\end{tabular}

Tableau 6 : Représentations de l'articulation des activités agricoles au territoire dans la convention territoriale du Pays VGBVD Matrix of interactions between farming and rural development in the convention territoriale of the Pays VGBVD 
La chambre d'agriculture s'est organisée en secteurs géographiques correspondant grossièrement au découpage en Pays du département. S'ils regroupent au sein d'un " comité de développement» les agriculteurs locaux souhaitant y participer, ils n'incluent pas les autres acteurs locaux dans la réflexion. Le risque paraît donc exister de l'élaboration parallèle de projets agricoles plus ou moins reliés à la construction territoriale, sans autre point de ralliement qu'une rationalisation de l'utilisation de l'espace.

\section{Les Pays dans la constitution DE NOUVEAUX LIEUX DE RÉFLEXION ET DE DÉBAT POUR UNE AGRICUL- TURE MULTIFONCTIONNELLE?}

Dans ces trois Pays périurbains, nous trouvons dans les documents consultés et dans les entretiens menés, une reconnaissance de l'espace agricole et des activités, qu'ils portent comme une composante du territoire, voire dans certains cas comme un élément fort d'une identité du territoire en construction et qui peut contribuer à son attractivité.

La grille que nous proposons a effectivement permis d'en rendre compte de façon synthétique. Elle permet de visualiser des places différentes de l'agriculture dans la construction territoriale et les distinctions qui sont opérées entre productions au niveau de chacun des Pays. En effet, dans les trois cas, nous constatons que des activités agricoles du territoire sont différenciées et mises en avant de façon distincte. Il s'agit soit d'une production (cas de la viticulture), soit d'un regroupement sous une catégorie encore en construction d'activités et de productions diverses (cas de «l'agriculture durable» dans le Pays du Sud Toulousain). Le développement des circuits courts de commercialisation pour un approvisionnement local est évoqué dans les trois Pays, mais avec des contenus variés. L'agriculture dont on parle est donc celle qui peut faire sens au regard du projet de territoire parce qu'elle est à la fois une garante du cadre de vie privilégiant des espaces ouverts et un fournisseur potentiel de productions au service de la consommation locale ou de l'attractivité du territoire. Mais, les territoires de projets ne semblent pas actuellement porteurs d'une approche globale et intégrée de la place de l'agriculture sur le territoire.
Ces résultats sont en accord avec les observations produites autour d'autres villes dans la même période, qui soulignent également que seules certaines productions, ou certains systèmes de production sont susceptibles d'apporter des services à la ville (Bertrand et Rousier, 2010). Par rapport à cette vision «tertiaire » de l'agriculture (Martin, 2010), cette grille permet de différencier quelles dimensions de la construction territoriale sont visées par les services que pourraient rendre l'activité agricole.

On peut se demander à la suite de ces observations comment "l'agriculture conventionnelle », c'est-à-dire autour de Toulouse, mais, également dans l'est du Tarn, les grandes cultures sur des surfaces d'exploitation étendues, va s'articuler avec ces projets de territoire. Nous avons vu en effet que, bien qu'elles soient dans tous les cas majoritaires en surface (tableau 3), ces productions ne sont que difficilement prises en compte par les Pays. C'est en effet surtout à travers la procédure SCoT qu'elles sont intégrées, par le biais de l'objectif de réduire la consommation d'espace agricole malgré des prévisions de croissance démographique. En HauteGaronne, cet objectif de moindre consommation est désormais inscrit de façon chiffrée, ce qui est nouveau, dans le plan agricole départemental établi en 2008, mais peine à se traduire encore sous forme cartographique. Dans le Tarn, notamment dans le Pays étudié, cet objectif est inscrit dans le SCoT.

Dans les deux départements, nous observons l'attention portée par les chambres d'Agriculture à ces documents de planification territoriale, attention qui s'est traduite par la création de services en charge du suivi de l'aménagement du territoire. La participation des chambres d'Agriculture à la construction d'un projet agricole articulé à un projet plus global du territoire est moins apparente, alors même que ces deux chambres d'Agriculture ont maintenu des conseillers agricoles locaux, regroupés dans des antennes géographiques locales. Ces organismes s'appuient classiquement sur une légitimité élective, qui est reconnue, développent leur position d'experts, qui leur permet d'exercer des prestations de service dans l'action d'aménagement (diagnostics agricoles notamment), mais peinent peut-être à mettre en œuvre le troisième type de légitimité identifié par S. Connelly et al. (2006), la légitimité des pairs, auprès des producteurs qui sont $a$ priori identifiés comme les plus à même de participer au pro- 
jet de territoire périurbain. De nouvelles coalitions sont peut-être donc en train d'apparaître, associant nouvelles structures territoriales et acteurs, jusquelà marginalisés, du développement agricole, voire de nouveaux acteurs, autour de nouvelles figures encore en construction, comme celle « d'agriculture durable » que nous avons rencontrée dans le Pays du Sud Toulousain.

\section{Conclusion}

Cette diversité d'acteurs et de partenariats entre acteurs, peut renvoyer à différentes articulations entre activité agricole et construction territoriale. L'usage de la grille de lecture que nous présentons ici peut fournir une vision synthétique des différents positionnements de la question agricole et permettre d'identifier des formes négligées d'articulation aux dynamiques territoriales. En ce sens, elle pourrait constituer un outil d'animation territoriale.

\section{Bibliographie}

Agnew J., 1990. Les lieux contre la sociologie politique, EspacesTemps, $n^{\circ} 43-44$, p. 87-94.

Barreau M., Taulelle F., 2008. Le département du Tarn et les territoires de projets, Revue du Tarn, n² 209, p. 1-11.

Bertrand N., Rousier N., 2010. Le rapport de l'agriculture à la ville : vers quelles proximités économiques? dans BERTRAND
N. (dir.), L'agriculture dans la ville éclatée, Grenoble, Cemagref, p. 47-64.

Connelly S., Richardson T., Miles T., 2006. Situated legitimacy: Deliberative arenas and the new rural governance, Journal of Rural Studies, vol. 22, n³, p. 267-277.

Demazière D., Dubar C., 1997. Analyser les entretiens biographiques. L'exemple des récits d'insertion, Nathan, Paris, $350 \mathrm{p}$.

Di Meo G., 1998. Géographie sociale et territoires, , Paris, Nathan $320 \mathrm{p}$.

Duvernoy I., 2009. Le conseil agricole public dans l'aire urbaine toulousaine entre développement sectoriel et territorial, dans Compagnone C., Auricoste C., Lemery B. (dir.), Conseil et développement en agriculture : quelles nouvelles pratiques?, Paris, QUAE/Educagri, p. 153-164.

Frenot S., 2009. Toulouse, moteur de la forte poussée démographique en Midi-Pyrénées, INSEE Midi-Pyrénées, nº 116 , $6 \mathrm{p}$.

Hajer M. A., 2004. A frame in the fields. Policy making and the reinvention of politics, dans Hajer M. A., WagenaAr H. (dir.), Deliberative policy analysis. Understanding governance in the network society, Cambridge, Cambridge University Press, p. 88-110.

Hajer M. A., Wagenaar H., 2004. Deliberative policy analysis. Understanding governance in the network society, Cambridge, Cambridge University Press, 307 p.

Martin S., 2010. Multifonctionnalité de l'activité agricole ou multi-usage de l'espace : vers une tertiarisation de l'agriculture, dans Bertrand N. (dir.), L'agriculture dans la ville éclatée, Grenoble, Cemagref, p. 65-76. 